\title{
Design and fabrication of runaway probe for studying the behavior of runaway electrons in IR-T1 Tokamak
}

\author{
Taher Afsari ${ }^{1} \cdot$ Mahmood Ghoranneviss $^{1} \cdot$ Sakineh Meshkani ${ }^{1} \cdot$ Mohammad Reza Ghanbari $^{2}$
}

Received: 4 October 2019 / Accepted: 26 December 2019 / Published online: 28 January 2020

(c) The Author(s) 2020

\begin{abstract}
Several theoretical and experiential studies of runaway electrons in the well-known Tokamaks of the world have been made over the past few decades. In the present study, the measurements of runaway electrons energy were carried out by a new runaway probe in the IR-T1 Tokamak.In the IR-T1 Tokamak, the hard X-ray diagnostic system is used as a diagnostic global measurement system at the vessel wall of Tokamak. Runaway probe was first designed and fabricated for local measurement near the plasma edge in IR-T1 Tokamak. The new diagnostic has the advantage that the local interaction of runaways with materials can be evaluated, along with the direct measurement of the runaway electrons. The runaway probe consists of 2 LYSO (Lu 1.8Y.2SiO5:Ce) crystals covered by a graphite housing which is shielded by tungsten filter placed in the direction of runaway electrons. The main elements of the runaway probe are LYSO crystals which convert the energy of runaway electrons into visible light which is instructed to silicon photomultipliers. The present study aimed to evaluate a new runaway probe in the IR-T1 Tokamak to detect beta and gamma rays. The results indicated that electron spectrum is between $500 \mathrm{keV}$ and $2 \mathrm{MeV}$ at the plasma edge in the IR-T1 Tokamak.
\end{abstract}

Keywords Tokamak $\cdot$ Runaway electron energy $\cdot$ Runaway probe $\cdot$ Beta and gamma rays

\section{Introduction}

The basic reason for studying superthermal electrons is frequently defined by the great heat flux coming to plasma coating materials with a runaway current created during a major Tokamak plasma disruption. In this case, the large heat flux produced can cause damage to the walls of large fusion machines such as JET, JT60-U, especially in ITER [1]. Applying the toroidal electric field to Tokamak plasma leads to an increase in the velocity of the thermal electrons. The collision forces between the electron decrease rather than increasing at a faster rate if the relative velocity between electrons and ion species exceeds the electron thermal velocity. However, it is clearly regarded as an unstable situation. In fact, the electrons run away if the electric field is fixed, and the velocity of electrons increases from the critical

Mahmood Ghoranneviss

ghoranneviss@gmail.com

1 Plasma Physics Research Center, Science and Research Branch, Islamic Azad University, Tehran, Iran

2 Department of Basic Sciences, Garmsar Branch, Islamic Azad University, Garmsar, Iran speed $v_{\text {cr }}$ The required electric field for a given electron velocity to be $v_{\text {cr }}$ is called the Dreicer field $[2,3]$. This generation mechanism is called "primary generation process." Another process which can put electrons into the runaway region of velocity space arises from close collision between already existing runaway electrons and thermal electrons (an avalanche mechanism). Although test particles in plasma are mainly affected by the same large-impact parameter collision with small momentum transfer, the smaller number of close collisions which transfer larger momentum is more important in this process. In this case, the relevant close collisions are considered as those lifting a slow electron above the critical velocity of runaway. In addition, generation in this process requires the presence of primitive runaway electrons with high energy. Thus, the avalanche mechanism plays a significant role in large-scale Tokamaks [4].

The energy and current of these electrons depend on some factors such as plasma density, plasma voltage, temperature and plasma impurities, and magnetohydrodynamic activity (MHD). For example, runaway electrons are produced in the $\mathrm{MeV}$ range due to low density or high loop voltage at the start of the plasma or the main rupture (plasma startup [5-7], disruptions [8-11], or instabilities [12, 13]). Further, 
runaway electrons with such high energy can damage the Tokamak wall. Based on the reports, up to $700 \mathrm{MJ}$ of energy can be transferred to the wall by the runaway electrons in the ITER [14]. Therefore, the assessment of the methods involved in terminating and suppressing the runaway electron beam is very important [15].

A large number of studies have centralized on runaway electrons in the plasma core and the vessel wall although no measurement has been made at the plasma boundary. Furthermore, various techniques have been used in the vessel wall. For example, hard X-ray radiation measurements are done at the time of discharge when the high-energy runaway electrons collide with plasma ions and the limiter/wall of Tokamak [16-18] leading to Bremsstrahlung radiation, which results in generating Hard X-ray photons. However, further studies are necessary to evaluate various aspects of the runaway electrons such as electron cyclotron emission (ECE) and Bremsstrahlung radiation [19] in order to obtain more information about runaway electrons. "Experimental setup" section describes the IR-TI Tokamak and the experimental setup. "Results and discussion" section presents the results, and discussion is elaborated in "Conclusion" section.

\section{Experimental setup}

IR-T1 is an ohmically heated and small research Tokamak located at the Plasma Physics Research Center (PPRC). Tokamak IR-T1 is an air-core Tokamak without a shell of copper. It has major radius of $R=45 \mathrm{~cm}$ and a minor radius of $a=12.5 \mathrm{~cm}$, the plasma current $I_{\mathrm{p}}=20-35 \mathrm{KA}$, the toroidal magnetic field is $B_{\mathrm{t}}=0.7-0.8 \mathrm{~T}$, the line average electron density is $\tilde{n}=(0.7-1.5) \times 10^{19} \mathrm{~m}^{-3}$ and the electron temperature is $T_{\mathrm{e}}=10-500 \mathrm{eV}$. The main parameters of IR-T1 Tokamak are given in Table 1. The desired Tokamak consists of a vacuum vessel with a circular cross section that was made from a stainless steel welding structure with two toroidal breaks and a minor radius, $b=15 \mathrm{~cm}$.

In the present study, a new probe (runaway p) was designed and fabricated to spectral measurements of runaway electrons. After initial tests, it was installed on

Table 1 The main parameters of IR-T1 Tokamak

\begin{tabular}{ll}
\hline Parameters & Value \\
\hline Major radius & $45 \mathrm{~cm}$ \\
Minor radius & $12.5 \mathrm{~cm}$ \\
Toroidal field & $<1.0 \mathrm{~T}$ \\
Plasma current & $<40 \mathrm{KA}$ \\
Discharge duration & $<35 \mathrm{~ms}$ \\
Electron density & $0.7-1.5 \times 10^{19} \mathrm{~m}^{-3}$ \\
Toroidal field coils & 16 \\
\hline
\end{tabular}

IR-T1 Tokamak and ${ }^{90} \mathrm{Sr}(546 \mathrm{keV}, 2.28 \mathrm{MeV})$ source was employed simultaneously in order to calibrate new probe. Beta particles are fast electrons (or positrons) emitted as a result of the decay of a neutron (or proton) in nuclei which contain an excess of the respective nucleon. Nuclides that decay directly to the ground state are "pure beta emitters". ${ }^{290} \mathrm{Sr} /{ }^{90} \mathrm{Y}$ is such a source, emitting a continuous spectrum of fast electrons up to a maximum energy of $0.546 / 2.27 \mathrm{MeV}$. The diameter of the source is $14 \mathrm{~mm}$. ${ }^{90} \mathrm{Sr}$ is a beta emitter, with a half-life of 28.8 years, decaying into ${ }^{90} \mathrm{Y}$ with an end-point energy of $0.546 \mathrm{MeV} .{ }^{90} \mathrm{Y}$ is also a beta emitter, with a half-life of $64 \mathrm{~h}$ and an endpoint of about $2.3 \mathrm{MeV}$. Due to the half-lives of the two isotopes, a secular equilibrium between the two decay processes is reached, with a continuous beta spectrum which extends up to $2.3 \mathrm{MeV}$ and has two components associated with the two isotopes. The measurements were taken with a ${ }^{90} \mathrm{Sr} /{ }^{90} \mathrm{Y}$ radioactive beta source (which added the complication of a beta spectrum with two components), and by the use of a simple Geiger counter to detect the electrons after they traverse a sheet of material. While the basic measurements and the evaluation of a rough value of the mass absorption coefficient formed the basis for all common activities, additional measurements and detailed GEANT simulations were then carried out for a further understanding of the obtained results. The Geiger was connected to a control box, which incorporates a variable power supply and a digital scaler, to record the counts from the detector. A Geiger counter is usually operated in the "plateau" region, i.e., with an applied voltage which minimizes the variations in the output signal amplitude (hence in the number of registered counts) as a function of the voltage. The plateau curve was measured for the counter employed in the present experiment registering the counts due to the presence of the radioactive source, placed at a fixed distance from the detector, for increasing values of the bias voltage. A value of $300 \mathrm{~V}$ was then chosen, roughly corresponding to the center of the plateau curve. Due to the fact that the calibration was carried out at the Science and Technology Park in Tehran and since I was Phd student at another university, they refused to give me the Beta rays spectrum and gave me only the calibration results.

The influence of less energetic electrons on the produced signal resulted in creating an error in the spectral measurements of about $20 \%$. The results of the probe were tested with an error of approx $150 \mathrm{keV}$, which is related to the amount of energy lost through the graphite sheath due to the type of graphite used. This probe includes two scintillating LYSO ( $\mathrm{Lu} \mathrm{1.8Y.2SiO5:Ce)} \mathrm{crystals} \mathrm{[20],} \mathrm{which} \mathrm{are}$ connected to silicon photomultipliers (Micro Fc 30035). The probe is located in the direction of runaway electrons in IR-TI Tokamak. The light output of the first scintillator 
is a function of the runaway electron energy, which is proportional to the number of incident runaway electrons. The probe aims to develop a tool which provides information of the runaway electron behavior. Figure $1 \mathrm{a}$ and $\mathrm{b}$ displays schematic [18] and real photographs of runaway probe.

The outside of the runaway probe is covered by $1.5-\mathrm{mm}$ graphite housing. The graphite housing shields the detectors from the low energy particles. Only runaway electrons with an energy exceeding $500 \mathrm{keV}$ can pass through the graphite [21]. The second crystal is separated by a piece of $2.5 \mathrm{~mm}$ tungsten. The electron and gamma beams cross graphite sheath and reach the first crystal, while the tungsten filter causes only the gamma to pass through and reach the second crystal. Thus, based on the difference in the signals obtained from the first and second crystals, the signal is obtained based on the beta signal. As illustrated in Fig. 1b, the crystals are white in order to reflect better and faster the light inside the crystal and direct toward the silicon photomultipliers. In addition, optical grease is used as a vertical retainer at the interface between the crystals and the silicon photomultipliers. It is worth noting that the refractive index of the grease should be greater than the crystals and less

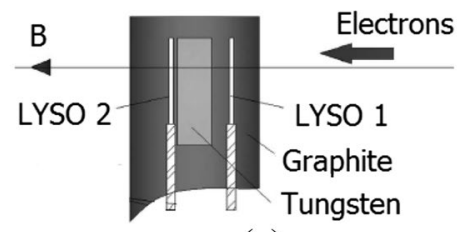

(a)
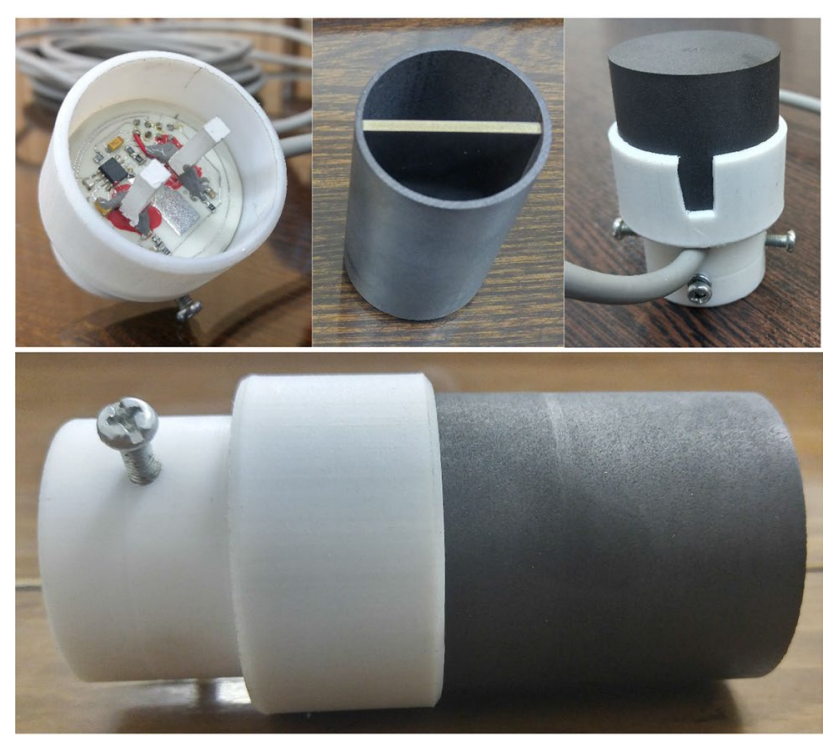

(b)

Fig. 1 a The schematic of runaway probe in IR-T1 Tokamak demonstrates the position of LYSO crystals inside the graphite housing. The second crystal is shielded by $2.5 \mathrm{~mm}$ tungsten and $\mathbf{b}$ the real photos of runaway probe than the silicon photomultipliers. Then, the overall reflection does not occur, and the light can easily pass through and reach the silicon photomultipliers. Simple simulation of the energy loaded on the graphite part of the probe by considering the mass attenuation coefficient of carbon graphite of beta particles can be made under certain conditions including an active cross section of the probe $S=2 \times 10^{-4} \mathrm{~m}^{2}$, and thickness of $d=0.0015 \mathrm{~m}$. Electrons with energy higher than $500 \mathrm{keV}$ lost about $E_{\text {abs }} \approx 150 \mathrm{keV}$ in the graphite part.

The crystals encompass some characteristics such as $2760{ }^{\circ} \mathrm{C}$ melting point of $20 \mathrm{~mm}$ length, $1 \mathrm{~mm}^{2}$ cross section, $45 \mathrm{~ns}$ decay time, $420 \mathrm{~nm}$ peak emission, and 27600 photons/MeV light output [20]. The crystal length was selected to have enough signal.

\section{Results and discussion}

In IR-T1 Tokamak, electrons can be accelerated up to a maximum energy of $2 \mathrm{MeV}$ [22]. In addition, the absorbed energy in the crystal per one electron (gamma) passing through the crystal is considered as a slowly varying function of the electron (gamma) energy between $500 \mathrm{keV}$ and $2 \mathrm{MeV}$. The deposited energy in the crystal is about $50 \mathrm{keV}$ (10 keV) per one electron (gamma). In order to gain information about runaway electrons behavior in the energy range between $500 \mathrm{keV}$ and $2 \mathrm{MeV}$, signals from the first and second crystal should be used. In the present study, discharge time was $32 \mathrm{~ms}$, the runaway probe located at the tangent to the limiter in all discharge, and the value of vertical magnetic field was $2.75 \mathrm{kV}$. Figure $2 \mathrm{a}-\mathrm{f}$ shows the time evolution of the plasma current, loop voltage, the signal of crystal 1 , the signal of crystal 2 , beta signals, and Mirnov oscillation, respectively. Based on the results, the maximum plasma current is around $20 \mathrm{KA}$. As illustrated in Fig. 2f, the most magnetic instability occurs between 24 and $28 \mathrm{~ms}$ due to these instabilities. Further, as shown in Fig. 2a, the plasma current decreases during this period and the plasma is off. As observed in Fig. $2 \mathrm{c}$ and d, the minimum amount of radiation is observed at the given time interval by decreasing the density and plasma current. The spectral analysis of the obtained signals occurs when the first crystal can detect and measure electrons with energy above $500 \mathrm{keV}$.

The total absorbed energy in 3D GEANT4 [21], simulation by first and second crystals, see Fig. 3, reduces proportionally with increasing thickness of the tungsten.

The minimum amount of energy measured by the first crystal is $500 \mathrm{keV}$. Therefore, the runaway probe can resolve electron with energies between $500 \mathrm{keV}$ and $2 \mathrm{MeV}$. To obtain the mean energy of runaway electrons in shorter time intervals, the discharge time of $50 \mathrm{~ms}$ was broken down to 10 equal time intervals of $5 \mathrm{~ms}$ each [22]. It was observed that after $15 \mathrm{~ms}$ from the plasma initiation, the photons with 

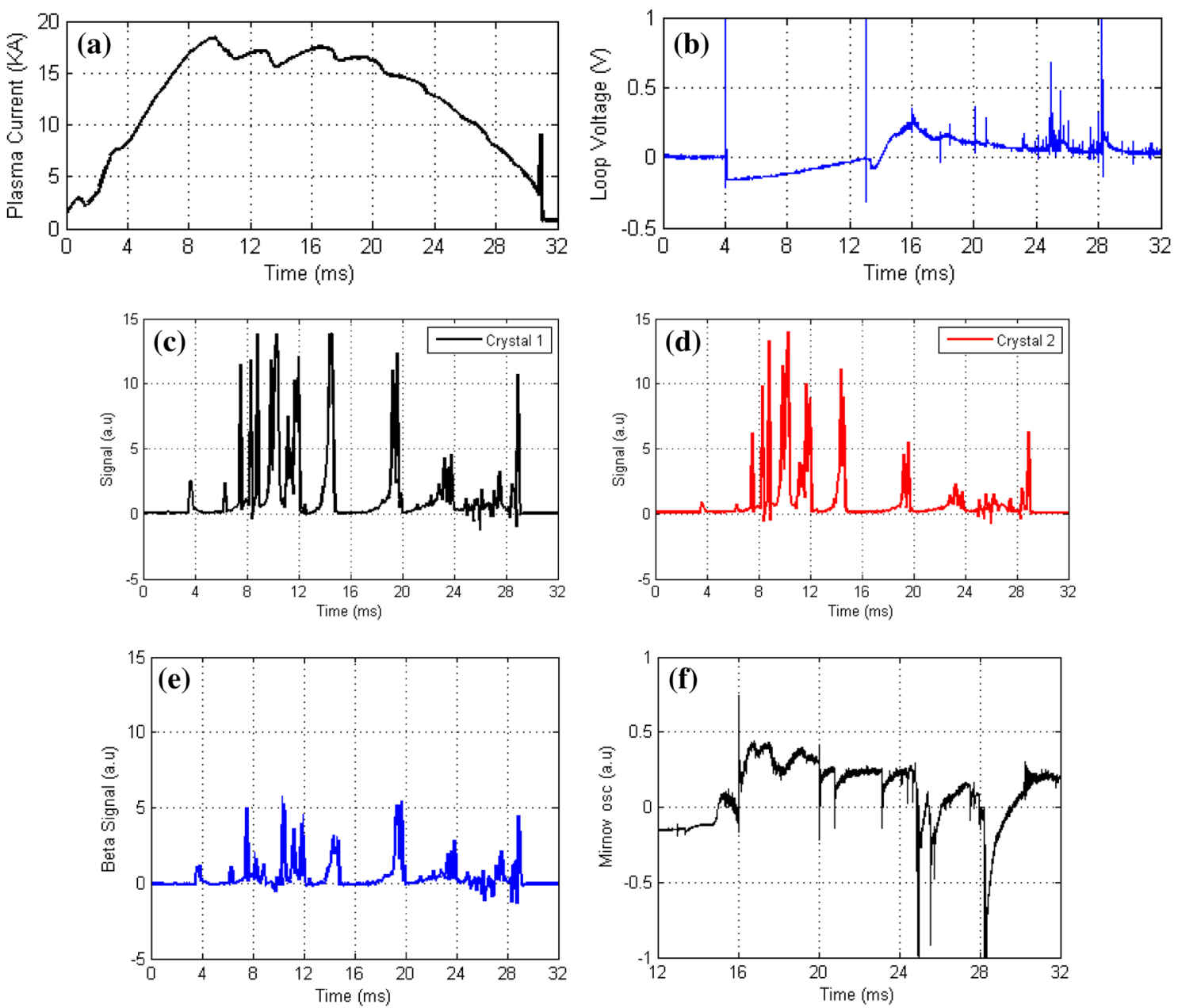

Fig. 2 Time evolution of the a plasma current, $\mathbf{b}$ loop voltage, $\mathbf{c}$ signal of crystal 1, $\mathbf{d}$ signal of crystal 2, e beta signals and $\mathbf{f}$ Mirnov oscillation

the energy more than $100 \mathrm{keV}$ are starting to appear. These photons grow longer in count until the plasma fade away. In this investigation, the time interval of $50 \mathrm{~ms}$ was chosen due to the fact that the hard X-ray spectra could be detected even after the plasma had died out. It can be seen from the hard $\mathrm{X}$-ray count graphs that the number of low energy photons increases as the plasma moves toward the end of its existence. According to HXR measurements, we will have the highest amount of energy in the interval of 20-30 ms. In the 50-ms interval, the amount of energy measured by the HXR is between $100 \mathrm{keV}$ and $1 \mathrm{MeV}$, which is consistent with what was measured by the runaway probe in that interval.

\section{Conclusion}

In the present study, runaway electrons were measured by generating runaway probe. In IR-T1 Tokamak, the hard $\mathrm{X}$-ray diagnostic system was used as a diagnostic global system, and accordingly, this diagnostic system was utilized to obtain a modest amount of information about runaway electrons. Then, the new diagnostic was used to measure runaway electrons directly near the plasma edge of IR-T1 Tokamak for the first time. In addition, the runaway probe was used as a local diagnostic system in IR-T1 Tokamak. Further, runaway probe was first implemented to access the information at the edge of the plasma. The results indicated that the values of beta and gamma rays are measured in the range of $500 \mathrm{keV}-2 \mathrm{MeV}$ with an approximate tool error of $150 \mathrm{keV}$ for the runaway probe. Finally, comprehensive information related to the behavior of runaway electrons can be obtained by combining the information obtained from the 

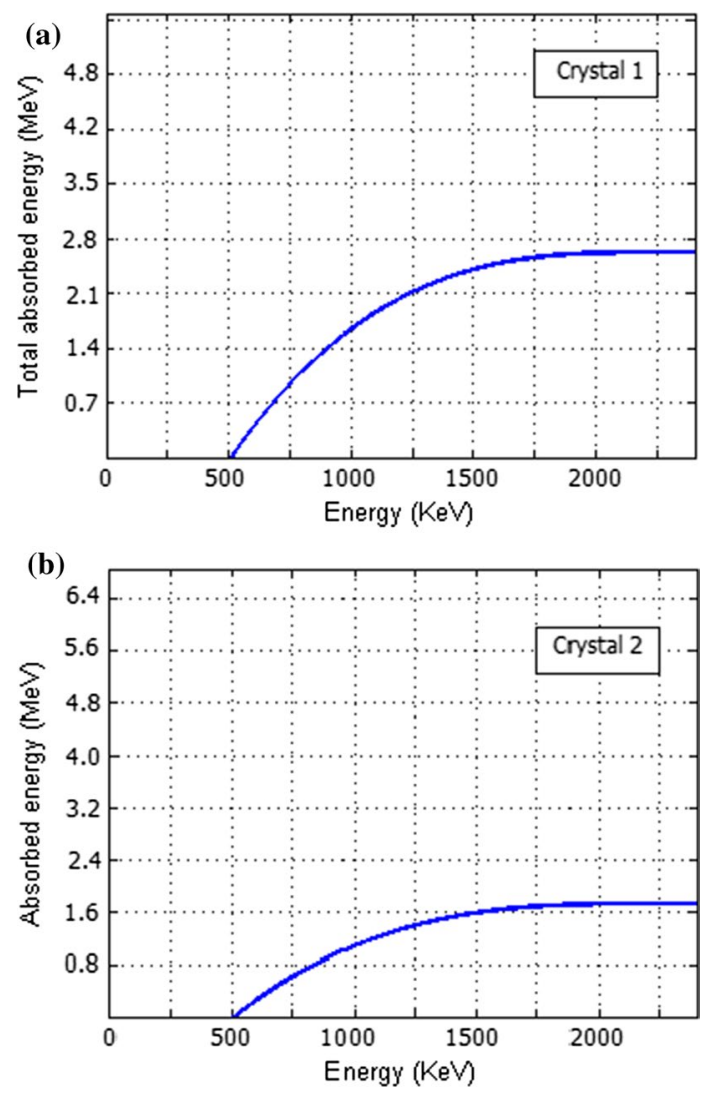

Fig. 3 3D Monte Carlo GEANT4 code simulation of the total absorbed energy by each crystal in the probe

Hard X-ray at the vessel wall of Tokamak and runaway probe near the plasma edge in IR-T1 Tokamak.

Open Access This article is licensed under a Creative Commons Attribution 4.0 International License, which permits use, sharing, adaptation, distribution and reproduction in any medium or format, as long as you give appropriate credit to the original author(s) and the source, provide a link to the Creative Commons licence, and indicate if changes were made. The images or other third party material in this article are included in the article's Creative Commons licence, unless indicated otherwise in a credit line to the material. If material is not included in the article's Creative Commons licence and your intended use is not permitted by statutory regulation or exceeds the permitted use, you will need to obtain permission directly from the copyright holder. To view a copy of this licence, visit http://creativecommons.org/licenses/by/4.0/.

\section{References}

1. Federici, G., Brooks, J.N., Coster, D.P., Janeschitz, G., Kukuskhin, A., Loarte, A., Pacher, H.D., Stober, J., Wu, C.H.: Assessment of erosion and tritium codeposition in ITER-FEAT. J. Nucl. Mater. 1(290), 260-265 (2001)

2. Dreicer, H.: Electron and ion runaway in a fully ionized gas. I. Phys. Rev. 115(2), 238 (1959)

3. Miyamoto, K.: Fundamentals of plasma physics and controlled fusion. National Inst. for Fusion Science (2011)
4. Sokolov, I.U.: Multiplication' of accelerated electrons in a Tokamak. JETP Lett. 20(29), 218-221 (1979)

5. Finken, K.H., Watkins, J.G., Rusbüldt, D., Corbett, W.J., Dippel, K.H., Goebel, D.M., Moyer, R.A.: Observation of infrared synchrotron radiation from Tokamak runaway electrons in TEXTOR. Nucl. Fusion 30(5), 859 (1990)

6. Jaspers, R.E.: Relativistic runaway electrons in Tokamak plasmas. Proefschrift Technische Universiteit Eindhoven-Meteen Samenvatting in het Nederlands (1968). ISBN 90-386-0474-2

7. Jaspers, R., Finken, K.H., Mank, G., Hoenen, F., Boedo, J.A., Cardozo, N.L., Schuller, F.C.: Experimental investigation of runaway electron generation in TEXTOR. Nucl. Fusion 33(12), 1775 (1993)

8. Gill, R.D.: Generation and loss of runaway electrons following disruptions in JET. Nucl. Fusion 33(11), 1613 (1993)

9. Helander, P., Eriksson, L.G., Andersson, F.: Runaway acceleration during magnetic reconnection in Tokamaks. Plasma Phys. Controlled Fusion 44(12B), B247 (2002)

10. Eriksson, L.G., Helander, P., Andersson, F., Anderson, D., Lisak, M.: Current dynamics during disruptions in large Tokamaks. Phys. Rev. Lett. 92(20), 205004 (2004)

11. Plyusnin, V.V., Riccardo, V., Jaspers, R., Alper, B., Kiptily, V.G., Mlynar, J., Popovichev, S., de La Luna, E., Andersson, F.: Study of runaway electron generation during major disruptions in JET. Nucl. Fusion 46(2), 277 (2006)

12. Parail, V.V., Pogutse, O.P.: The kinetic theory of runaway electron beam instability in a Tokamak. Nucl. Fusion 18(3), 303 (1978)

13. Savrukhin, P.V.: Generation of suprathermal electrons during magnetic reconnection at the sawtooth crash and disruption instability in the T-10 Tokamak. Phys. Rev. Lett. 86(14), 3036 (2001)

14. Putvinski, S., Barabaschi, P., Fujisawa, N., Putvinskaya, N., Rosenbluth, M.N., Wesley, J.: Halo current, runaway electrons and disruption mitigation in ITER. Plasma Phys. Controlled Fusion 39(12B), B157 (1997)

15. Yoshino, R., Tokuda, S., Kawano, Y.: Generation and termination of runaway electrons at major disruptions in JT-60U. Nucl. Fusion 39(2), 151 (1999)

16. Esposito, B., Solis, R.M., Van Belle, P., Jarvis, O.N., Marcus, F.B., Sadler, G., Sanchez, R., Fischer, B., Froissard, P., Adams, J.M., Cecil, E.: Runaway electron measurements in the JET Tokamak. Plasma Phys. Controlled Fusion 38(12), 2035 (1996)

17. Esposito, B., Martın-Solıs, J.R., Poli, F.M., Mier, J.A., Sanchez, R., Panaccione, L.: Dynamics of high energy runaway electrons in the Frascati Tokamak Upgrade. Phys. Plasmas 10(6), 2350-2360 (2003)

18. Kudyakov, T., Finken, K.H., Jakubowski, M.W., Lehnen, M., Xu, Y., Schweer, B., Toncian, T., Van Wassenhove, G., Willi, O., TEXTOR tokamak: Spatially and temporally resolved measurements of runaway electrons in the TEXTOR Tokamak. Nucl. Fusion 48(12), 122002 (2008)

19. Finken, K.H., Abdullaev, S.S., Jakubowski, M.W., Jaspers, R., Lehnen, M., Schlickeiser, R., Spatschek, K.H., Wingen, A., Wolf, R., TEXTOR tokamak: Runaway losses in ergodized plasmas. Nucl. Fusion 47(2), 91 (2007)

20. https://www.crystals.saint-gobain.com

21. Agostinelli, S., Allison, J., Amako, K.A., Apostolakis, J., Araujo, H., Arce, P., Asai, M., Axen, D., Banerjee, S., Barrand, G.2., Behner, F.: GEANT4 - a simulation toolkit. Nucl. Instrum. Methods Phys. Res. Sect. A: Accel. Spectrom. Detect. Assoc. Equip. 506(3), 250-303 2003

22. Hasanvand, N., Riazifar, M.R., Alipour, R., Meshkani, S., Ghoranneviss, M.: Improving plasma confinement by controlling hard X-ray. Chin. Phys. Lett. 33(11), 115202 (2016)

Publisher's Note Springer Nature remains neutral with regard to jurisdictional claims in published maps and institutional affiliations. 